\title{
LOCALIZED BILHARZIAL GRANULOMA OF THE LUNG SIMULATING A TUMOUR

\author{
BY
}

\author{
S. H. EL MALLAH AND M. HASHEM
}

From the Thoracic Surgery Unit and the Department of Experimental Medicine, Kasr El Aini (Cairo)

(RECEIVED FOR PUBLICATION AUGUST 12, 1952)

The diagnosis of solitary, circumscribed lung shadows is one of the most difficult problems in thoracic surgery. Many of these cases are asymptomatic and are detected by mass radiography. The long list of conditions that lead to these shadows, and the fact that they share, to a great extent, the same signs and symptoms, show how difficult it may be to reach a definite diagnosis. Husfeldt and Carlsen (1950) stated that they had had to do thoracotomies on 33 occasions in order to diagnose such cases. It is not the intention of this paper to discuss the differential diagnosis of such shadows, but to report an unusual case.

One of us (S. H. M.) came across a patient with symptoms presenting mainly as a solitary lung shadow. Every possible method of investigation failed to clarify the diagnosis. Exploratory thoracotomy was therefore carried out, and, as the condition was judged to be carcinoma at operation, pneumonectomy was performed. On pathological examination of the specimen it turned out to be a case of bilharzia. To our knowledge a case of this type has not been reported before.

\section{CASE RePORT}

B. A. was a man aged 47 years who worked as a farmer and was seen in the Out-patient Department on December 31, 1950, complaining of pain in the right sid: of the chest accompanied by cough and slight expectoration for nine months. He had had two attacks of haemoptysis, but streaking of the sputum was a commoner event. At no time during his illness $h{ }_{1} d$ he any temperature, nor was the sputum foul.

The patient was well built. His pulse was 80 to the minute and regular. There was no pyrexia and the blood pressure was $120 / 80 \mathrm{~mm}$. Hg. He was not dyspnoeic and there was no clubbing of the fingers.

Except for an area of dullness and diminished air entry postero-laterally situated between the sixth and the eighth nios and corresponding to the position of the pain, there was otherwise nothing clinically abnormal in the chest.

He brought with him a postero-anterior radiograph of the chest which showed a circumscribed, rounded shadow about $6 \mathrm{~cm}$. in diameter in the middle zone of the right lung field. He said that this shadow in the radiograph had been diagnosed by his private doctor $\vec{\infty}$ as a lung abscess, for which he had been given peni- 0 cillin and streptomycin, but he felt no improvement.

He was admitted to hospital for further investigation. $\vec{c}$

In hospital further radiographs were made. Figs. $1 \subseteq$ and 2 are postero-anterior and lateral radiographs of the chest. They show a shadow in the middle zone, $\overrightarrow{0}$ and in the lateral view it lies in the subapical part of of the lower lobe below the major oblique fissure. Screening showed that the shad Jw was intrapulmonary, moving with the lung during respiration.

The sputum was repeatedly negative for Koch's bacillus. It was also examined by direct smears and paraffin sections for malignant cells, but none could be $\mathbb{D}$ detected.

The blood picture was: $\mathrm{Hb} 80 \%$; red cells, $5,190,000$; white cells, 8,600 (eosinophils $12 \%$, basophils $0 \%$, polymorphs $70 \%$ (nucleated staff cells $10 \%$ and segmented cells $60 \%$ ), lymphocytes $14 \%$, monocytes $4 \%$ ).

Such a degree of eosinophilia is not uncommon in Egypt, and especially in those patients where parasitic $\mathrm{O}$ infestation is common, but the urine and faeces of our $\stackrel{\oplus}{x}$ patient were negative for any parasitic infection.

The complement fixation test for hydatid disease was 3 also performed, but was negative. No further attention

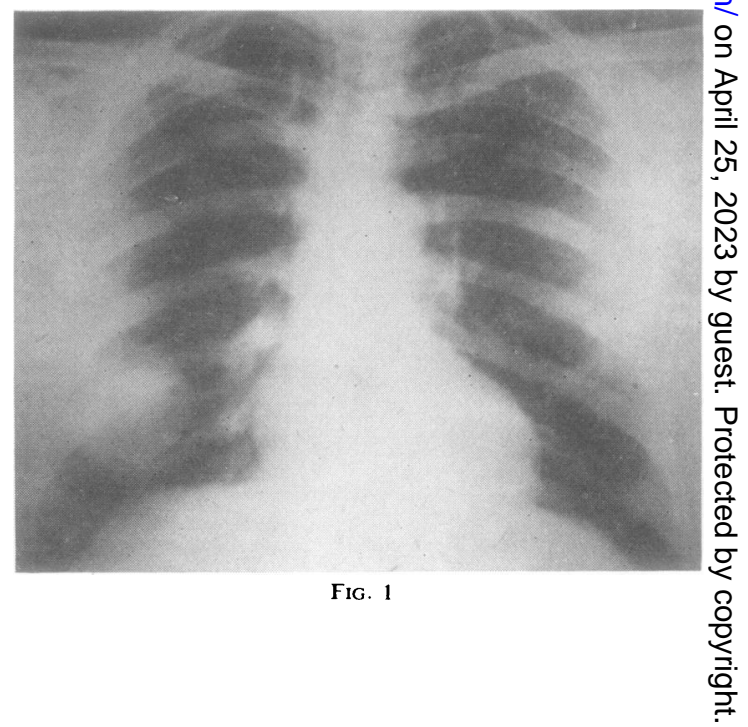




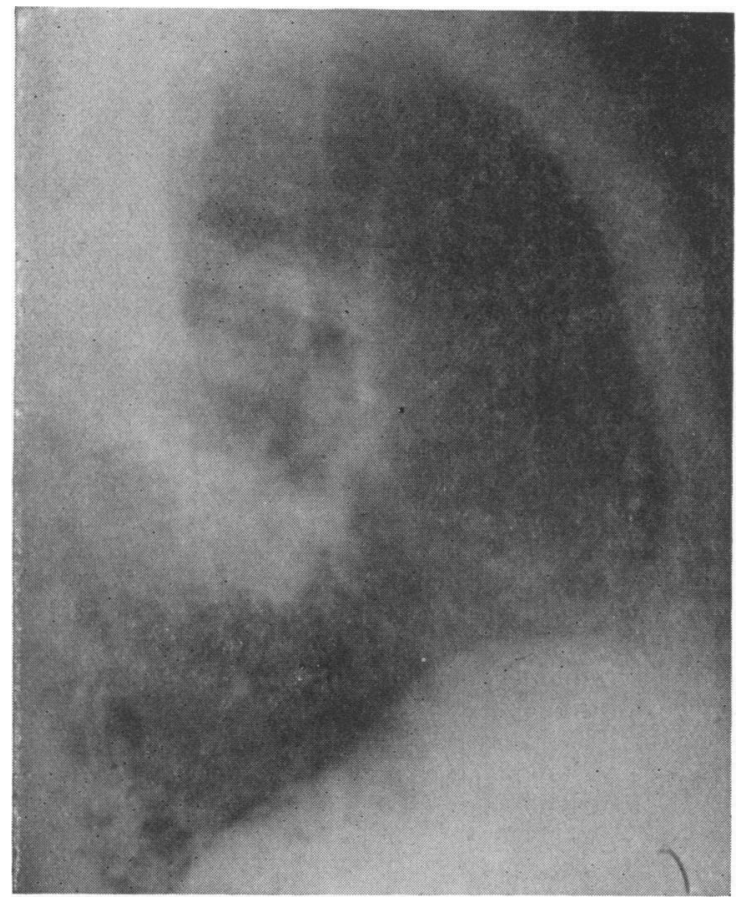

FIG. 2

was paid to the eosinophilia, and we now wonder if it would be helpful in the diagnosis of other cases.

Bronchoscopy was not done, because the patient had had two attacks of haemoptysis, and as the shadow was peripheral we expected very little information to be gained from it.

The possibility that the shadow might be a solitary secondary was thought of, but examination revealed no suspicion of a primary tumour.

The possibility of tumour, and especially cancer of the bronchus, could not be excluded, and exploratory thoracotomy was considered to be the next and remaining procedure for diagnosis and treatment.

The operation was performed on January 24, 1951. Under endotracheal gas and oxygen supported by pentothal and curare the chest was opened through the bed of the fifth rib.

The lower lobe was found to be atelectatic and the seat of a hard, indurated, big "tumour." This mass was firmly adherent to the chest wall; the rest of the lower lobe was adherent to the chest wall by thin, easily separable adhesions especially on the diaphragmatic surface. The oblique fissure was obliterat $\in \mathrm{d}$. The upper and middle lobes looked normal. The hilum was examined and no enlarged glands could be detected. The mass looked very much like a peripheral carcinoma, and, taking into consideration the age and the history of the patient, this diagnosis seemed to be almost certain. The adherent part was dissected off the chest wall extrapleurally and a dissection pneumonectomy performed. The patient stood the operation well and he passed through convalescence safely. He was discharged on March 16, 1951.

Since the operation he has been seen repeatedly and he is in perfect health, not dyspnoeic, and able to work moderately hard.

ExAmination of THE ExcIsed LUNG.-The excised lung was inflated with formalin and sectioned 10 days later. The upper and lower lobes appeared normal except for some thickening of the pleura, especially on the mediastinal surface. The transverse and the oblique fissures were obliterated by fibrous adhesions. The lung surface over the tumour showed a depression corresponding to the site of the tumour. The pleura over the mass was thickened and showed dense, vascular adhesions. The cut surface of the lower lobe (Fig. 3) revealed a mass which was more or less spherical (aboui $5 \mathrm{~cm}$. in diameter). It was surrounded by a fibrous capsule about $1 \mathrm{~mm}$. thick. The remaining part of the lower lobe was atelectatic and consolidated.

Histopathological Examination.-Sections from blocks selected from different parts of the lung lesion and the neighbouring tissues showed a fibrous thickening of the overlying pleura, which contained a few scattered bilharzial pseudo-tubercles, and some blood vessels presenting endarteritis obliterans. The lung tissue underlying the pleura presented patchy atelectasis and.

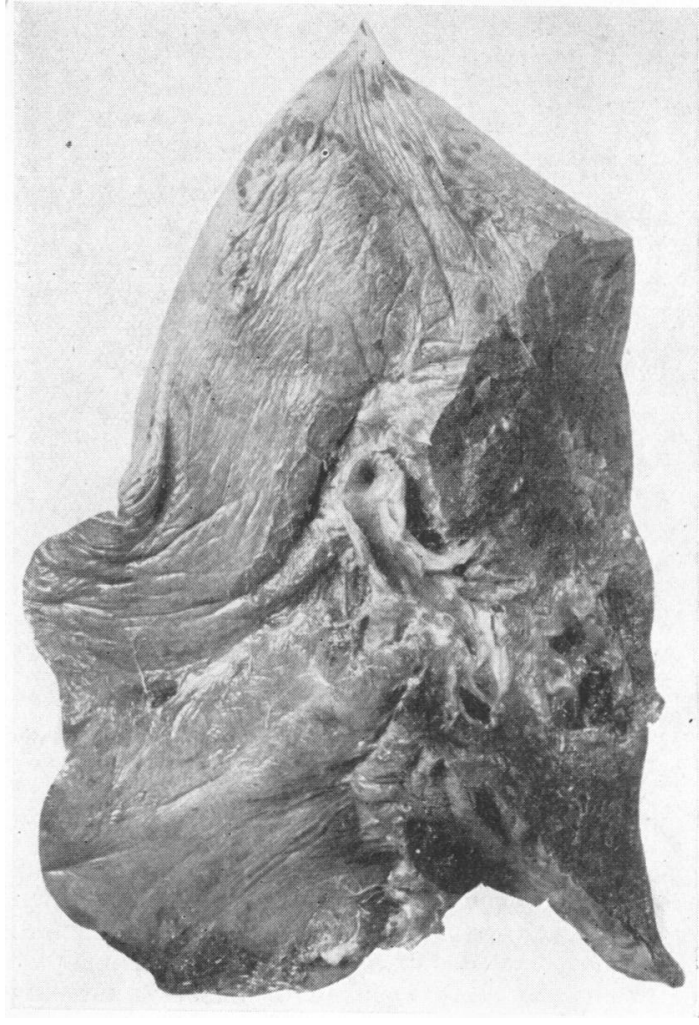

FiG. 3 


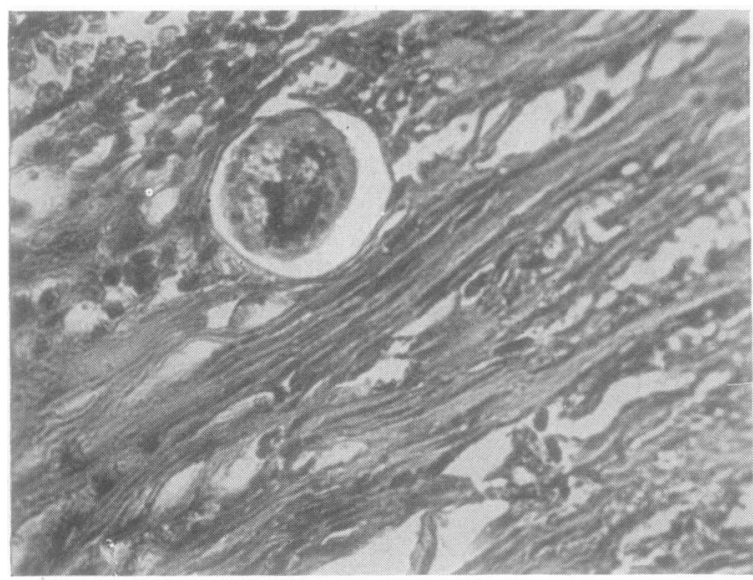

FIG. 4.-The broad zone of dense hyaline fibrous tissue with a degenerate ovum embedded in it $(-350)$

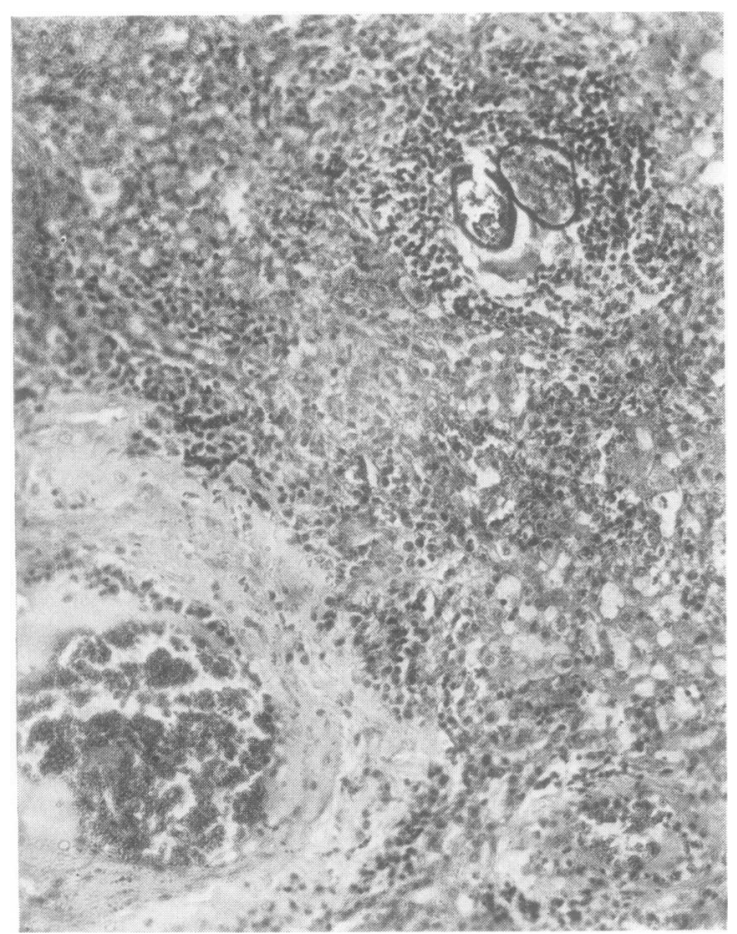

FIG. 5.-Dense infiltration by bilharzial granulation tissue. There is a degenerate ovum in the specific inflammatory reaction. A thick-walled vessel with perivascular fibrosis is seen in the corner. The rest of the section shows atelectatic lung tissue $(\therefore 180)$.

compensatory emphysema associated with interstitial fibrosis; a few bilharzial pseudo-tubercles were found in the parenchyma and each was formed of a centrally placed degenerated bilharzia ovum or the remnants of an ovum, commonly engulfed by one or more large foreign body cells and surrounded by a cellular reaction consisting mainly of histiocytes and eosinophils and

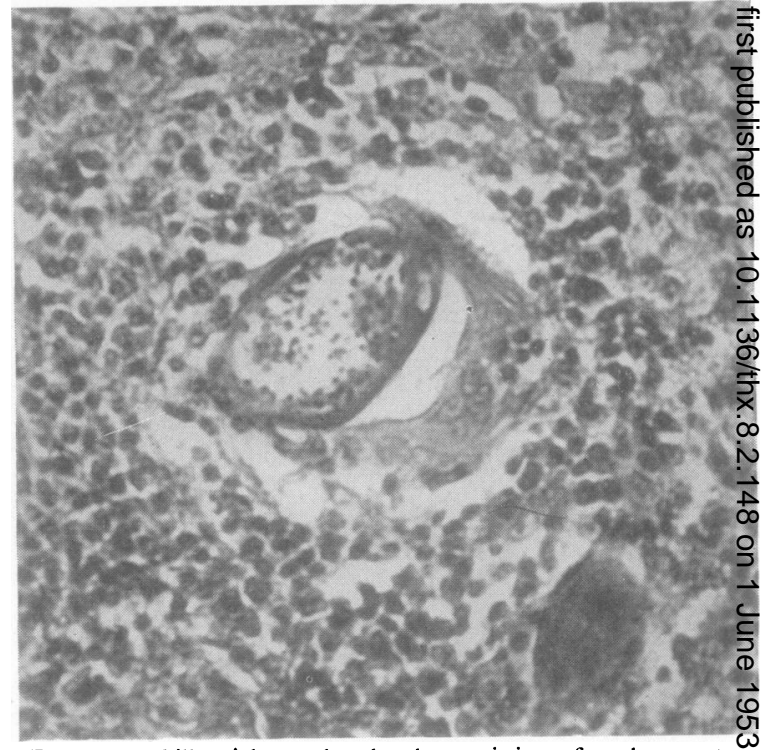

FIG. 6.-A bilharzial pseudo-tubercle consisting of a degenerate calcified ovum engulfed by a large foreign body giant cell and $\square$ surrounded by granulation tissue rich in eosincphilia and 0 histiocytes $(3350)$.

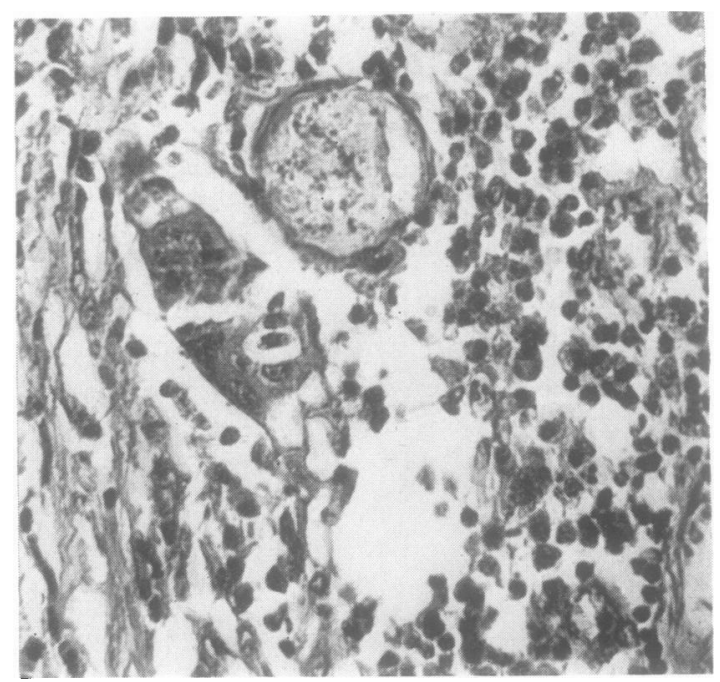

FIG. 7-Two degenerate ova in the acute inflammatory exudate in the innermost layer bordering the broken-down centre $(\therefore 350)$.

fibroblasts. The amount of eosinophilia was inversely proportionate to the degree of the fibroblastic reaction. Next to this zone of atelectasis there was chronic inflammatory fibrosing granulation tissue more or less replacing the lung tissue with some collapsed inflamed bronchioles containing inflammatory exudate rich in eosinophilia, together with some few bronchiectatic cavities. Bilharzial pseudo-tubercles were also found in this zone and the vessels showed endarteritis obliterans. 


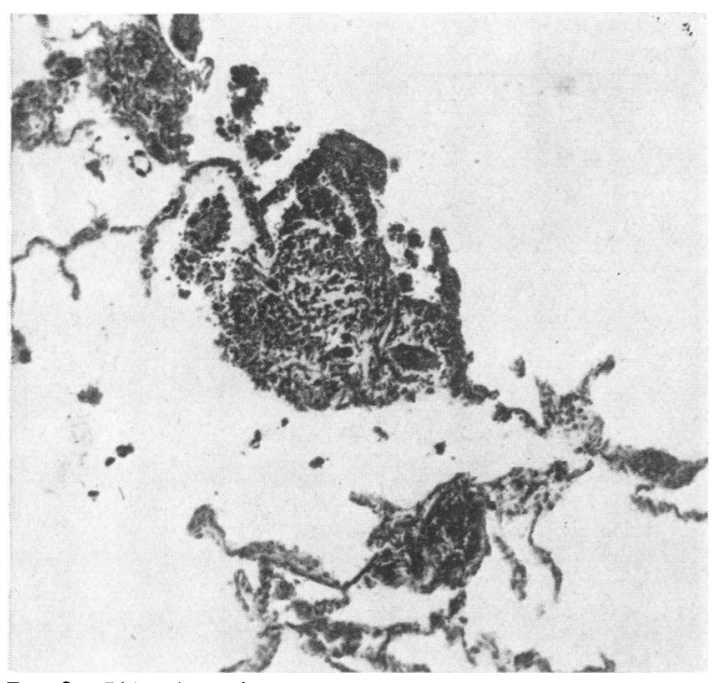

FIG. 8.-Bilharzioma in the apparently healthy part of the lung. Note the degenerate ovum with the surrounding bilharzial granulation tIssue in the interstitial tissue of the lung $(x 180)$

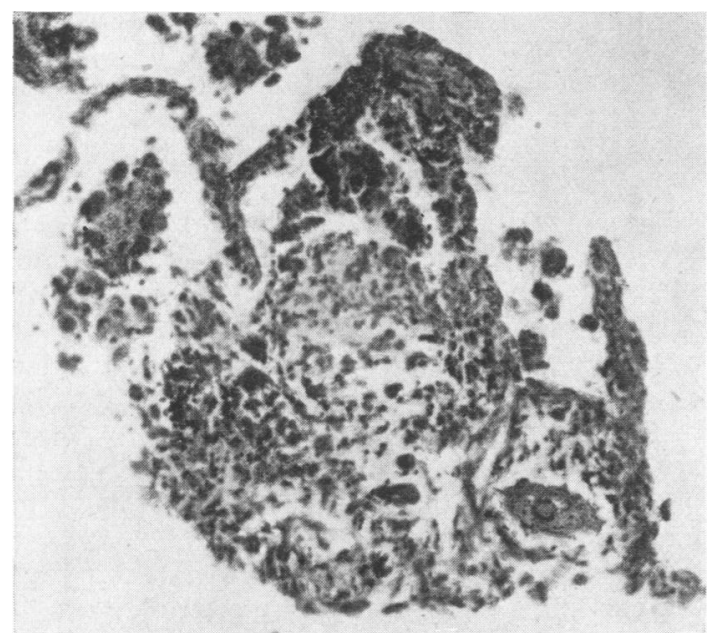

FIG. 9.-High-power view of Fig. $8 \times 350$ ).

Outside this there was a zone of an acute inflammatory cellular exudate, rich in eosinophils, with a few necrotic bilharzia ova scattered among the inflammatory cells. This zone formed the part of the mass nearest to the hilum. Sections from other parts of the lung showed a few bilharzial pseudo-tubercles and endarteritis obliterans affecting the blood vessels. The accompanying photomicrographs were taken from different parts of the tumour (Figs. 4, 5, 6, 7, 8, and 9).

\section{Discussion}

This case raises several points for discussion. Is the mass bilharzial, or is it a non-specific, non- inflammatory process occurring in a bilharzial lung? What are the possibilities of correct diagnosis of similar cases? What is the correct treatment if the diagnosis of such cases were possible?

With regard to the first question, we believe that the pathological findings are in favour of the essential factor in the production of this mass being bilharzia. The greater number of bilharziomata in the site of the mass as compared to other parts of the lung suggests a heavier infestation by embolized ova in this region. The eosinophilic fibroblastic reaction among the various layers of the mass is in favour of a chronic bilharzial process. The acute inflammatory reaction in the central part of the mass can be explained by hypersensitivity to local liberation of toxins which may have been enhanced by anti-bilharzial treatment, and/or secondary infection with non-specific micro-organisms in an area relatively devitalized by heavy bilharzial infestation.

With regard to the possibility of diagnosis of similar cases, it seems that a certain diagnosis is very unlikely. According to Professor Erfan, Professor of Tropical Medicine, Kasr El Aini (Cairo), examination of the sputum is only valuable if it shows bilharzia ova. Although this may help to direct attention to the possibility, it cannot exclude the possibility that a carcinoma coexists with bilharzia. There are recorded cases where bilharzia ova were found among carcinomatous tissue in the lung.

Regarding treatment of these cases, we feel that it is unlikely that anti-bilharzial treatment would affect such a lesion, taking in consideration the lung and bronchial damage that had occurred as a result of the lesion. If it were possible to diagnose similar cases-and we think that frozen sections may help-then a more conservative resection, that is to say lobectomy, would be the ideal treatment.

\section{SUMMARY}

A rare case of solitary lung tumour which turned out to be the result of bilharzial infection is reported. The histopathology of the condition, the possible diagnoses, and the treatment are discussed.

We wish to thank Mr. Seoudi for preparation of the photomicrographs and Mr. Memdouh for preparing the radiographs.

REFERENCE

Husfeldt, E., cnd Carlsen, C. J. (1950). Thorax, 5, 229. 